\title{
ON-LINE MEASUREMENT OF PARTICLE SIZE AND COMPOSITION
}

\author{
O. Kievit, J.C.M. Marijnissen, P.J.T. Verheijen and B. Scarlett \\ Delft University of Technology, Faculty of Chemical Engineering and Materials Science, \\ Particle Technology Group, P.O. Box 5054, 2600 GA Delft, The Netherlands.
}

\begin{abstract}
The Particle Technology Group at Delft University of Technology is developing an instrument for online, real-time measurement of size and chemical composition of individual aerosol particles. Particles are sampled from an aerosol by using aerosol beam techniques. Experimental results and a theoretical model for particle beams have been used to design an improved beam generator. The apparatus comprises an exchangeable capillary nozzle and three vacuum chambers in series. Collimated by the beam system the particles enter the ionization chamber of a time-of-flight mass spectrometer, where they can be vaporized and ionized by the pulse from a Q-switched YAG laser. Analysis of the generated ions with the mass spectrometer provides information on the chemical composition of the particles. This paper reports the first results obtained with a new experimental setup, based on earlier work. Mass spectra were observed for a static target and particles of sodium chloride. The results were compared with spectra obtained with a LAMMA-500.
\end{abstract}

\section{KEYWORDS}

Chemical; instrumentation; single particle analysis; time-of-flight mass spectrometry.

\section{INTRODUCTION}

Aerosols continue to play an important part in numerous fields of science. Since substructures are still getting smaller in semiconductor industry, even sub-micron particles can cause a electronic circuit to fail. It is therefor of the utmost importance to prevent the emission of particles and to reduce their concentration as much as possible. One method to determine the origin of a particle is to measure its size and chemical composition. These properties can serve as a fingerprint of the source, and thus contribute to the reduction of particle emission. Aerosols are also involved in atmospheric processes. It is common knowledge that both natural and antropogeneous particles can play a major role in atmospheric chemistry, acting as catalyst or reactant. In order to gain insight in the processes occurring in our atmosphere it would be very helpful to have an instrument capable of measuring particle size and chemical composition in real time, on an on-line basis. Until now research on real time chemical analysis of particles has not led to the development of a commercially available instrument. Particles have been volatilized with a hot filament, miniature oven or laser beam; the fragments were analyzed with a quadrupole or focal plane mass spectrometer (Sinha et al., 1982, 1984, 1986). The Particle Technology Group at Delft University of Technology has started a program whereby this approach is improved and extended, and combined with real time particle size measurement (Marijnissen et al., 1988). Particles are sampled from an aerosol by using aerosol beam techniques. The size of the particles is measured through light scattering. The chemical composition is determined with laser-induced time-of-flight mass spectrometry. 


\section{EXPERIMENTAL}

The instrument as described by Marijnissen et al. (1988) consists of a number of components (figure 1). Particles are sampled into an aerosol beam, which forms when the aerosol expands through a nozzle into a vacuum chamber [1]. Because the particles have a much higher inertia than the gas molecules they continue to travel at straight lines, while most of the gas is removed by the vacuum pump. The aerosol beam generator was built using results obtained with a glass prototype (Kievit et al., 1990). Improvements on the new apparatus include an easily exchangeable nozzle, and fixation of nozzle and skimmers which makes alignment unnecessary. The dimensions of the nozzle and skimmers and the distances between them, were optimized using a model for aerosol beams, as well as the results reported by other investigators (Estes et al., 1983; Sinha et al., 1982). Furthermore the nozzle geometry has been changed to yield a smaller divergence angle. The divergence half angle for $1.87 \mu \mathrm{m}$ polystyrene particles is about $0.7^{\circ}$, which is smail compared to values measured by Estes (1983). It is expected that surrounding the aerosol with a sheath of filtered air will improve the sampling efficiency of the aerosol beam generator.

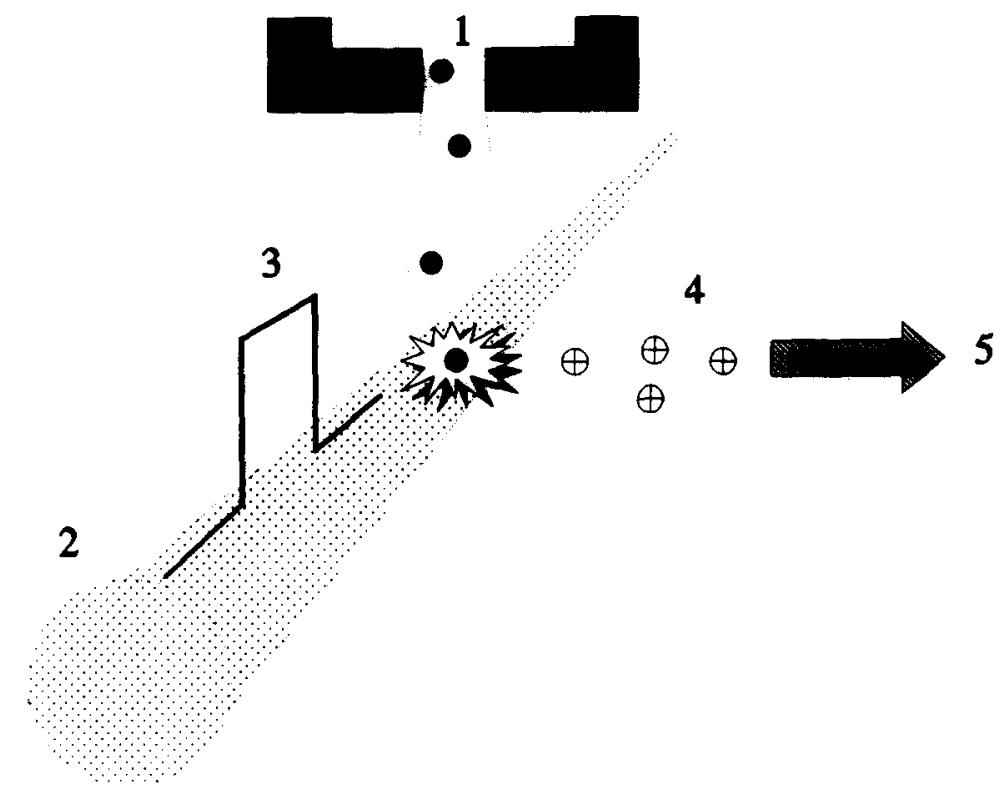

Fig. 1. Schematic representation of the instrument.

Collimated by the beam system the particles enter the ionization chamber of a time-of-flight mass spectrometer. In the finished instrument the aerosol beam will intersect with the beam of a helium-neon laser [2]. The light scattered by a particle will be measured to give an indication of the particle size. Furthermore the scattering signal will be used to trigger a second laser. This second laser is a Q-switched Nd:YAG laser (Lumonics Ltd., model HY1200). It is capable of generating pulses of $10 \mathrm{~ns}$ length, containing up to $1200 \mathrm{~mJ}$ of energy $(\lambda=1064 \mathrm{~nm})$. By using frequency doubling crystals the output can be converted into a multi-wavelength pulse, containing $450 \mathrm{~mJ}$ at the fundamental wavelength, $400 \mathrm{~mJ}$ at the second harmonic $(\lambda=532 \mathrm{~nm})$ and $100 \mathrm{~mJ}$ at the fourth harmonic $(\lambda=266 \mathrm{~nm})$ [3]. The described laser pulse vaporizes the particle and ionizes the fragments. An electric field accelerates the ions [4] into the flight tube of a time-of-flight mass spectrometer. The mass spectrometer, which has been developed at the 'Institut für Lasermedizin', at the University of Düsseldorf, detects the arrival of ions at the end of the flight tube [5]. The signal from the detector is recorded by a digital oscilloscope (LeCroy, model 9314M). The spectrum of flight times can be converted into a mass spectrum, which provides information on the chemical composition of the particle. 
Up till now the YAG laser has been fired at a fixed repetition rate, without prior detection of the particles. In all experiments an acceleration voltage of $2 \mathrm{kV}$ was used. It was found that the ion formation was at an optimum when the laser pulses contained $7 \mathrm{~mJ}$ of energy at the primary wavelength, $6 \mathrm{~mJ}$ at the second harmonic, and $2 \mathrm{~mJ}$ at the fourth harmonic. The laser output was focused with a positive lens with a focal distance of $250 \mathrm{~mm}$, to yield a spot size of approximately $2 \mathrm{~mm}$. The resulting power density is in the order of $10^{7} \mathrm{~W} / \mathrm{cm}^{2}$, which is low as compared to the power density used in the LAMMA-500 (about $10^{\circ} \mathrm{W} / \mathrm{cm}^{2}$ ). The mass spectrometer was calibrated with various alloys. In the first experiments a static target was fixed in the centre of the ionization chamber and analyzed. The target was manufactured by pressing sodium chloride into a tablet. In other experiments aerosol particles were analyzed. An aerosol was generated by nebulizing a $0.8 \%$ solution of sodium chloride. In order to dry the particles the aerosol was passed through a diffusion drier and a heated glass tube.

\section{RESULTS AND CONCLUSIONS}

The mass spectrum from the sodium chloride tablet, shown as figure $2 \mathrm{~b}$, contained primarily $\mathrm{Na}^{+}$-ions. When sodium chloride is analyzed with a LAMMA-500, an instrument with a similar operating principle, heavier ions are also found in the spectrum (figure 2a). However, the LAMMA-500 uses only the fourth harmonic of the YAG radiation. It is likely that the combination of infrared and ultraviolet radiation we used in our experiments causes more extreme fragmentation as compared to just ultraviolet. Therefor larger ions can not be found in the spectrum.
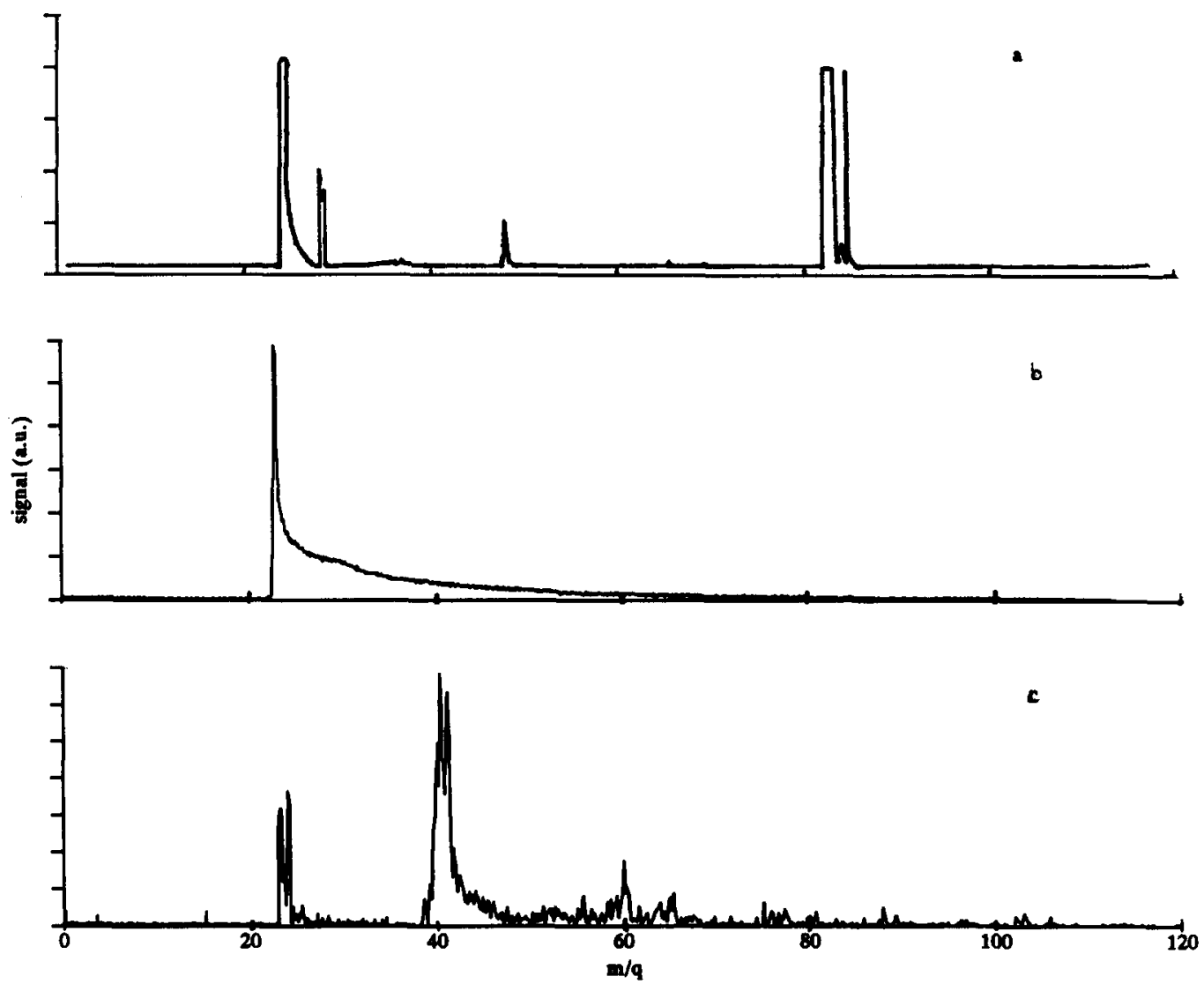

Fig. 2. Positive mass spectra from sodium chloride. The upper spectrum (2a) was measured with a LAMMA-500 (Marijnissen et al., 1988). The other spectra were recorded from a static target (2b), and a particle from the aerosol beam (2c). 
In the second set of experiments we tried to analyze aerosol particles. Figure $2 \mathrm{c}$ shows one of the mass spectra we obtained by firing the YAG laser at a fixed repetition rate of $10 \mathrm{~Hz}$, and monitoring the signal from the detector plates. As in the spectrum from the static target, a signal is found at $m / q=23$. However, there are also peaks at $\mathrm{m} / \mathrm{q}=41$ and $\mathrm{m} / \mathrm{q}=59$. We think that these peaks represent hydrated sodium ions. If the droplets from the nebulizer do not completely dry there will still be water present in the particles when entering the aerosol beam generator. According to Dahneke (1974) the evaporation of droplets in an aerosol beam is inhibited by cooling effects caused by the gas expansion. It is therefor possible that a particle hit by a laser pulse still contains a considerable amount of water, which enables the formation of hydrated ions.

The hypotheses made in this paper will be verified in the near future by measuring the spectra from other inorganic salts. Future work will focus on the implementation of light scattering equipment in the measurement cycle to size the particles and trigger the YAG laser, and further development of the chemical analysis.

\section{ACKNOWLEDGEMENTS}

The authors would like to thank the Netherlands Foundation for Chemical Research (SON) for supporting the investigations. Financial aid was provided by the Netherlands Organization for Scientific Research (NWO) and the Foundation for Technical Sciences (STW).

\section{REFERENCES}

Dahneke, B.E. (1974). In: Rarefied gas dynamics (K. Karomcheti ed.), pp. 197-203. Academic Press, New York.

Estes, T.J., V.L. Vilker and S.K. Friedlander (1983). Characteristics of a capillary-generated particle beam. J. Coll. Interface Sci., 23, 84-94.

Kievit, O., J.C.M. Marijnissen, P.J.T. Verheijen and B. Scarlett (1990). Some improvements on the particle beam generator. J. Aerosol Sci., 21, Suppl, 1, 685-688.

Marijnissen, J., B. Scarlett and P. Verheijen (1988). Proposed on-line aerosol analysis combining size determination, laser induced fragmentation and time-of-flight mass spectroscopy. J. Aerosol Sci., 19, 1307-1310.

Sinha, M.P., C.E. Giffin, D.D. Norris, T.J. Estes, V.L. Vilker and S.K. Friedlander (1982). Particle analysis by mass spectrometry. J. Coll. Interface Sci., $\underline{87}, 140-153$.

Sinha, M.P. (1984). Laser-induced volatilization and ionization of microparticles. Rev. Sci. Instrum., 55 , 886-891.

Sinha, M.P. and S.K. Friedlander (1986). Mass distribution of chemical species in a polydisperse aerosol: measurement of sodium chloride in particles by mass spectrometry. J. Coll. Interface Sci., 112, 573-582. 